\title{
“蓝嘴唇”: 缺氧性肺动脉高压肺血管重构研究进展
}

\author{
王睿，潘进进，王定友，姬雨轫，刘明成，金海峰，袁予辉" \\ 大连医科大学肿瘤干细胞研究院, 大连 116044 \\ * 联系人, E-mail: yuhuiyuan@hotmail.com \\ 收稿日期: 2021-10-22; 接受日期: 2021-11-15; 网络版发表日期: 2021-12-16 \\ 国家自然科学基金项目(批准号: 81472492)资助
}

\begin{abstract}
摘要 缺氧性肺动脉高压是一种肺动脉压异常升高的综合征, 起因是长期慢性缺氧诱发肺动脉血管收缩和重构, 发病率高、生存期短, 尚无有效治疗方法, 是临床面临的巨大难题和挑战. 慢性炎症和氧化应激反应诱导的肺小 动脉血管重构是缺氧性肺动脉高压的典型病理学特征。本文针对肺动脉血管重构的病理生理学特点、病理生理 学发病机制、治疗现状及展望等方面的最新研究进展进行总结, 并讨论肺动脉血管重构的机制研究和相关治疗 药物研发进展, 为缺氧性肺动脉高压的治疗提供思路.
\end{abstract}

关键词肺动脉高压, 缺氧, 肺动脉血管重构, 炎症, 氧化应激

肺动脉高压(pulmonary hypertension, PH)是一种 复杂的进行性心肺系统疾病, 确切发病机制尚不清楚, 患者生存期有限，临床上暂无有效治愈手段，PH也被 认为是心血管领域的“癌症”. PH患者因长期缺氧所致 嘴唇呈现不同程度的蓝紫色, 被称为“蓝嘴唇”, 大部分 患者遭受过误诊经历，甚至无法得到确诊却因病情过 于严重而去世 ${ }^{[1]}$, 因此, 需要给予该疾病更多的重视与 关注.

依据《中国肺动脉高压诊断与治疗指南(2021 版)》, “pulmonary hypertension”名词定义为肺动脉高 压, 血流动力学定义为, 于海平面、静息状态下, 经右 心导管检测的肺动脉平均压 $\geq 25 \mathrm{mmHg}$. 临床上将 $\mathrm{PH}$ 分为 5 类, 其中肺部疾病和(或)低氧所致 $\mathrm{PH}$ 属于第 3 类, 长期慢性低氧是此类PH的重要特征和发病诱因. 缺氧 性肺动脉高压(hypoxic pulmonary hypertension, HPH)
不仅是PH中常见类型之一，也是阻塞性肺疾病、限制 性肺疾病、高原心脏病等多种心肺疾病发生发展中的 关键环节, 是临床面临的巨大难题和挑战 ${ }^{[2 \sim 5]}$. 肺动脉 血管重构(pulmonary vascular remodeling)在HPH的发 病机制中发挥重要作用，本文将从HPH肺血管重构的 病理学特点、病理生理学机制、治疗现状及展望四个 方面对HPH的研究进展作一综述.

\section{1 病理学特点}

缺氧性肺血管收缩(hypoxic pulmonary artery vasoconstriction)及肺动脉血管重构是HPH的两大典型病理 特征, 其过程主要发生于肺小动脉血管 $(<500 \mu \mathrm{m})^{[6,7]}$. 肺动脉血管壁有 3 层, 包括单层内皮细胞组成的内 膜、平滑肌细胞和弹力板组成的中膜以及成纤维细胞

引用格式: 王睿, 潘进进, 王定友, 等. “蓝嘴唇”: 缺氧性肺动脉高压肺血管重构研究进展. 中国科学: 生命科学, 2021, 51: 1637-1645 Wang R, Pan J J, Wang D Y, et al. "Blue lips": research progress in pulmonary vascular remodeling in hypoxic pulmonary hypertension (in Chinese). Sci Sin Vitae, 2021, 51: 1637-1645, doi: 10.1360/SSV-2021-0432 
(fibroblasts)组成的外膜. 血管重构过程涉及血管壁三 层的变化, 是细胞肥大、增生、炎症改变的结果, 影 响细胞分化、调亡、过度迁移，以及细胞外基质沉积 (图1).

\section{1 肺动脉内膜内皮细胞}

肺动脉血管内皮细胞(pulmonary artery endothelial cell，PAEC)是排列在血管内膜的单层扁平状细胞，作 为血流与肺动脉中、外膜之间屏障，能保护肺动脉中 层的平滑肌细胞及外层的成纤维细胞免受血液中各种 细胞因子、毒素的直接刺激作用. 同时兼具内分泌功 能，生成多种血管活性物质和细胞因子，调节血管舒 张及收缩 ${ }^{[8,9]}$. 正常生理状态下, PAEC被认为是遗传稳 定的“静止状态”，一旦被激活，将诱导疾病的发生，参 与疾病的进展.

肺动脉系统功能紊乱是 $\mathrm{HPH}$ 重要病理特征之一,
缺氧诱导的内皮细胞损伤是 $\mathrm{HPH}$ 肺动脉内皮功能障碍 发生的始动环节. 慢性缺氧破坏肺动脉血管内膜的完 整性，紊乱内皮屏障功能，血液中的各种刺激因子跨 过细胞内皮屏障直接作用于肺动脉的中、外膜, 刺激 肺动脉平滑肌细胞(pulmonary artery smooth cell, PASMC)和成纤维细胞异常增殖, 造成细胞外基质(extracellular matrix, ECM)过度沉积, 诱导肺动脉中膜及 外膜增厚 ${ }^{[10]}$; 缺氧还能促进内皮细胞分泌血管收缩因 子(例如内皮素-1,5-羟色胺等), 减少舒张因子(例如一 氧化氮NO、前列环素、心房钠尿肽等)的分泌，加剧 肺动脉血管的收缩程度, 造成肺动脉血管重构 ${ }^{[11,12]}$. 此外, 长期缺氧能直接激活内皮细胞, 导致PAEC过度 增殖, 内膜增厚, 管腔狭窄, 肺血管阻力增加, 肺动脉压 升高, 而肺动脉压的升高又能诱导细胞分泌大量细胞 外基质，促进肺血管的增厚、重构和狭窄，形成恶性 循环, 加重HPH的发生发展 ${ }^{[9]}$.
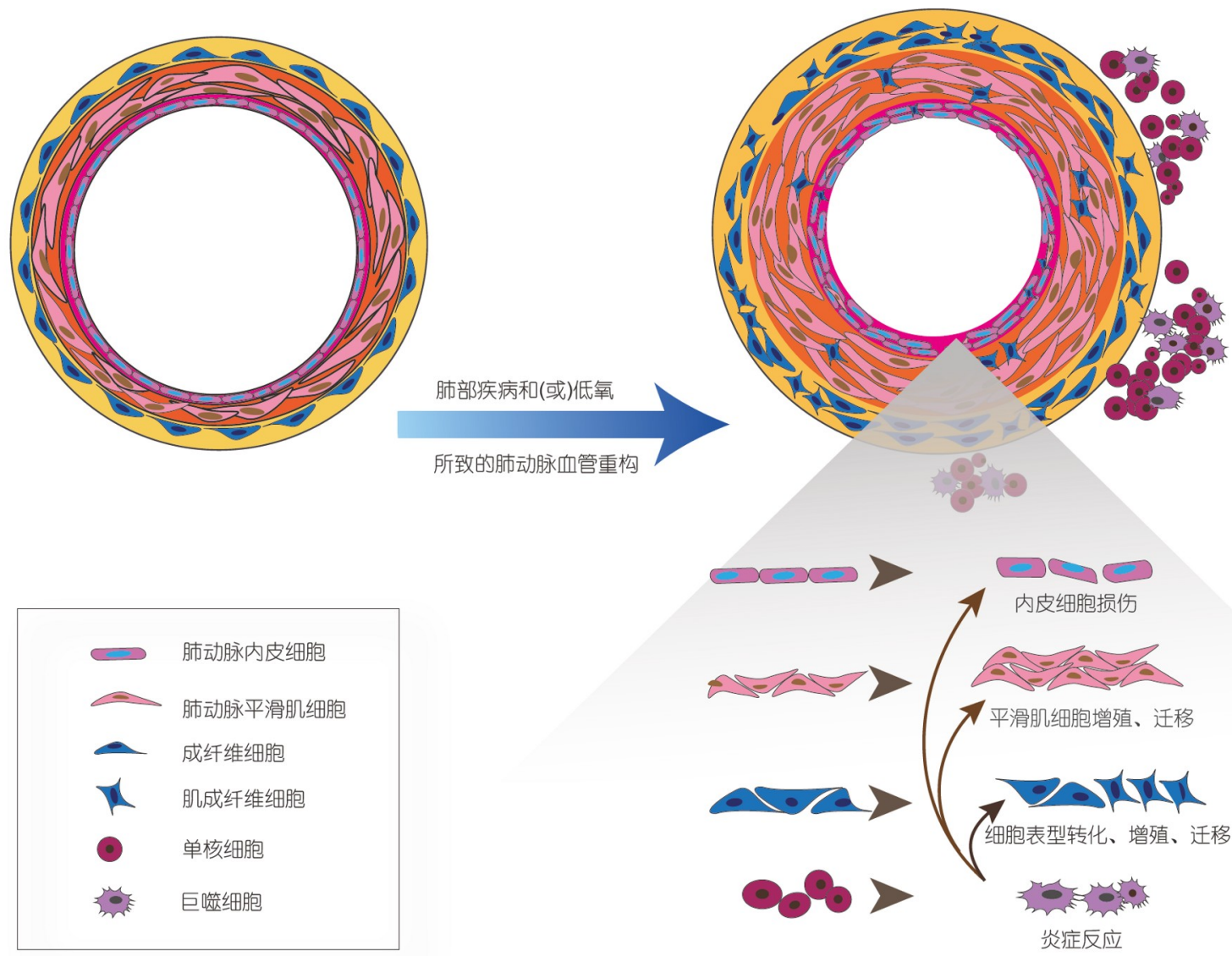

图 1 缺氧性肺动脉高压肺动脉血管重构病理特征示意图

Figure 1 Schematic diagram of pathological features of remodeled pulmonary arterioles in hypoxic pulmonary hypertension 


\section{2 肺动脉中层平滑肌细胞}

PASMC 是肺动脉血管中层的主要细胞, 是诱发肺 动脉血管重构的细胞学基础. 正常生理状态下, PASMC 的增殖与调亡处于动态平衡状态，成熟的 PASMC分化成为收缩表型. 病理情况下，各种生长因 子诱导PASMC发生去分化, 转化成为具有增殖、迁移 能力的合成表型. 远端肺微小动脉重构血管的PASMC 部分来源于收缩表型，去分化并迁移至远端小动脉血 管, 进行增殖和再分化等一系列细胞生物学活动, 参 与并促进HPH肺动脉血管重构与疾病发展 ${ }^{[13 \sim 15]}$.

大量研究广泛证实, PASMC的异常过度增殖在肺 动脉血管重构中处于主导性地位, HPH肺动脉血管中 层增厚的组织形态学标准是肥大和增生, 即PASMC体 积增大和数量增多 ${ }^{[16]}$. 慢性缺氧状态下, PASMC的增 殖与调亡比例失衡, 导致PASMC过度增殖, 最终造成 肺血管壁的不可逆性增厚、管腔狭窄甚至闭塞，以及 肺动脉压的持续升高 ${ }^{[17 \sim 20]}$. 已有研究报道, 生活在高 原(海拔高于 $3500 \mathrm{~m}$ )的居民与生活在海平面的居民相 比，远端肺动脉血管和小动脉中层的平滑肌细胞数量 增多 ${ }^{[21]}$. 缺氧诱导的PASMC的增殖性改变是导致缺氧 性肺动脉重构的重要环节, 探究PASMC过度增殖的潜 在确切分子机制, 应用有效策略进行针对性干预, 已经 成为近年来防治 $\mathrm{HPH}$ 的研究热点.

\section{3 肺动脉外膜纤维细胞}

成纤维细胞是构成肺动脉血管外膜的主要细胞, 其活化能造成外膜微环境的改变, 在HPH的发生发展 过程中具有重要作用 ${ }^{[22]}$. 缺氧条件下, 成纤维细胞对 低氧的感知及增殖反应较PASMC更为敏感，扮演“感 受器”角色率先被激活, 成纤维细胞迅速增殖并发生表 型转化为肌成纤维细胞(myofibroblast), 进而诱导肌成 纤维细胞从血管外膜向中膜、内膜迁移, 最终造成肺 小血管动脉血管壁的内、中、外膜呈现不同程度的结 构性增厚 ${ }^{[23,24]}$.

无肌型小动脉主要由内皮细胞和成纤维细胞组 成, 是肺循环中的重要容量型血管. 慢性缺氧条件下, 转化生长因子- $\beta$ 、血管紧张素 II 、凝血酶、内皮素和 纤连蛋白等表达增加, 诱导肺动脉外膜成纤维细胞的 胸腺细胞分化抗原(thymocyte differentiation antigen-1, Thy-1)启动子发生甲基化，Thy-1表达下调，成纤维细
胞表型转化为肌成纤维细胞, 进而诱发无肌型小动脉 肌化, 同时加速肌成纤维细胞增殖 ${ }^{[25 ~ 27]}$. 除了成纤维 细胞异常增殖外, ECM的沉积及胶原蛋白的交联重排 均能造成血管外膜增厚、血管硬度增加, 参与 $\mathrm{HPH}$ 进 展 ${ }^{[28]}$. 成纤维细胞还能招募巨噬细胞通过促炎信号转 导通路发生慢性炎症, 而巨噬细胞也能反过来促进成 纤维细胞产生胶原及生长因子, 诱导成纤维细胞增殖, 参与肺动脉血管重构 ${ }^{[29-31]}$.

\section{2 病理生理学机制}

\section{1 慢性炎症}

慢性炎症反应是诱发HPH疾病的极其重要机制之 一, 缺氧能造成肺动脉结构性与功能性的炎症反应发 生, 炎症能导致肺组织的氧气供需失衡, 形成局部低 氧微环境, 进一步加重机体的缺氧程度, 因此低氧(hypoxia)与炎症(inflammation)一直是研究者的关注热 点 ${ }^{[32,33]}$. 既往研究认为, HPH的血管炎症是以血管内膜 为中心, 由“内”向“外”进行, 近年来研究发现, 由成纤 维细胞启动炎症反应, 由“外”向“内”进行, 低氧条件下, 肌成纤维细胞分泌大量炎症因子(巨噬细胞趋化因 子、刺激因子、黏附蛋白), 诱导单核巨噬细胞、淋巴 细胞、树突状细胞等多种炎性细胞浸润, 导致肺小动 脉炎症的发生 ${ }^{[29,34,35]}$.

研究表明，剥脱掉外膜的肺动脉血管丧失激活巨 噬细胞的功能 ${ }^{[29]}$; 表观遗传修饰能够调控成纤维细胞 发生促炎表型转换, 其潜在机制可能与组蛋白去乙酰 化酶(histone deacetylase, HDAC)的表达升高及催化活 性增强相关 ${ }^{[30]}$; 炎性因子肿瘤坏死因子 $\alpha($ tumor necrosis factor $\alpha, \mathrm{TNF} \alpha$ ) 诱导PASMC转化成为促增殖和抗调 亡表型，白介素6(interleukin 6, IL-6)通过活化T细胞， 诱导巨噬细胞向M2型极化, 释放趋化因子CXCL12等 可溶性细胞因子进而促进PASMC的过度增殖, 参与肺 动脉中膜重构 ${ }^{[35]}$. HPH模型小鼠中, NALP3炎症小体 激活，IL-1 1 等促炎症细胞因子分泌增加，激活的炎症 细胞能进一步招募更多的炎症细胞, 释放大量的炎症 因子, 加剧炎症反应的发生，进而导致肺动脉内皮细 胞功能障碍，应用超氧化物歧化酶(superoxide dismutase, SOD)模拟物能有效缓解小鼠的HPH进展, 提示炎 症反应非独立存在, 与氧化应激的发生密切相关 ${ }^{[36]}$. 


\section{2 氧化应激}

活性氧(reactive oxygen species, ROS)族是指在生 物体内与氧代谢相关的含氧自由基，以及易形成自由 基的过氧化物的总称，主要包括超氧阴离子自由基、 脂氧自由基、羟自由基及过氧化氢 $\left(\mathrm{H}_{2} \mathrm{O}_{2}\right)$ 等. $\mathrm{ROS}$ 是 体内氧化代谢的重要分子, 线粒体及 $\mathrm{NADPH}$ 氧化酶 (nicotinamide vadenine dinucleotide phosphate oxidase, NOX)系统为其主要来源. 正常生理条件下, 机体精准 控制ROS的生成，参与细胞信号转导，调节信息传递. 慢性缺氧情况下，机体产生大量ROS，诱导氧化应激 (oxidative stress)反应，导致细胞内及细胞间信息传递 混乱, 诱发脂质过氧化, 损伤细胞正常生理功能, 参与 $\mathrm{HPH}$ 疾病进程 ${ }^{[3,37 \sim 41]}$.

已有研究证实，缺氧诱导的ROS过度生成能够造 成内皮细胞一氧化氮合酶(endothelial nitric oxide synthase, eNOS)发生解偶联反应，降低NO生成量 ${ }^{[42]}$. ROS作为第二信使在PASMC中直接或间接介导信号 转导通路, 例如P13K/Akt, MAPK/ERK, AMPK/STAT3 等, 导致PASMC过度增殖, 参与肺动脉血管重构 ${ }^{[40,41]}$. ROS能诱导内皮素-1、血栓素A2、前列环素等收缩血 管物质的释放, 导致肺动脉系统的持续收缩 ${ }^{\left[{ }^{8}\right]}$, 还能增 强缺氧诱导因子-1(hypoxia-inducible factor-1, HIF1)、核因子 $-\kappa \mathrm{B}($ nuclear factor kappa-B, NF- $\kappa \mathrm{B}) 、 \mathrm{AP}-1$ 等氧化还原敏感性转录因子的转录活性, 启动肺组织 的炎症反应, 促进HPH的发生与发展 ${ }^{[43]}$. 此外, 缺氧还 能降低机体内抗氧化酶的表达和(或)活性，包括调控 红血球核因子相关因子-2(nuclear factor erythroid-2 related factor 2, Nrf2)、SOD、硫氧还蛋白(thioredoxin, Trx)、谷胱甘肽(glutathione, GSH), 削弱ROS的清除作 用, 降低抗氧化系统的防御功能, 导致氧化应激水平增 加, 促进HPH的发生与发展 ${ }^{[44-47]}$. 因此, 如何有效清除 缺氧诱导产生的ROS成为HPH潜在治疗方法之一, 近 年来备受关注.

\subsection{HPH发生相关分子}

$\mathrm{HPH}$ 发生机制至今尚未阐明, 近年来, 国内外应用 HPH动物模型探究HPH发生发展机制的研究取得了一 定的进展. 尽管啮齿类动物模型(慢性低氧、慢性低氧 联合VEGF抑制剂等)不能完全模拟临床HPH的发病过 程, 但是对于研究信号转导通路探究发病机制具有重
要意义 ${ }^{[48]}$.

低氧诱导因子(hypoxia-induced factor, HIF) 是经典 的氧调节转录因子, 低氧条件下, 细胞内的HIF- $1 \alpha$ 蛋白 表达稳定, 是诱导PH发生的重要机制之一 ${ }^{[49,50]}$. 研究 报道，在慢性低氧诱导的条件下，平滑肌细胞特异性 敲除HIF- $1 \alpha$ 能延缓小鼠肺动脉血管重构和PH疾病进 程 ${ }^{[18]}$. 低氧条件下, HIF-1 $\alpha$ 能激活Ras相关区域家族 $1 \mathrm{~A}$ (Ras association domain family 1 A, RASSF1A)的转录, RASSF1A也能通过阻止HIF- $1 \alpha$ 的脯氨酰基化及蛋白 酶体降解, 增强HIF-1 $\alpha$ 的稳定性, 形成RASSF1A-HIF$1 \alpha$ 前馈环路, 从而增加糖酵解开关的活化, 驱动低氧信 号转导通路, 参与 $\mathrm{PH}$ 进展 ${ }^{[51]}$. 低氧条件下, CD146与 HIF- $1 \alpha$ 的表达相互促进, 形成低氧重编程信号轴, 促 进PASMC增殖、迁移，平滑肌细胞特异性敲除CD146 能够有效抑制慢性低氧诱导的小鼠肺动脉重构, 减轻 $\mathrm{PH}$ 症状 ${ }^{[20]}$.

2,6-二磷酸果糖激酶(phosphofructokinase-2/fructose-2,6-bisphosphatase, PFKFB)的亚型PFKFB3 是一 种酵解调节因子, 能促进糖降解, 参与细胞生存与增 殖. 研究发现, PAEC特异性敲除Pfkfb3 基因可显著降 低糖酵解水平, 减少糖丙酩酸的生成进而下调HIF- $2 \alpha$ 的表达，导致血小板源性生长因子 $\beta$ (platelet derived growth factor $\beta, P D G F \beta$ )、成纤维细胞生长因子 2 (fibroblast growth factor 2, FGF2) 以及CXCL12, IL-1 $\beta$ 等 促炎细胞因子表达减少, 抑制PASMC的过度增殖及 肺动脉血管周围的炎症细胞浸润，有效阻止 $\mathrm{HPH}$ 进 展 $^{[52]}$.

硒蛋白 $\mathrm{P}($ selenoprotein $\mathrm{P}, \mathrm{SeP})$ 广泛存在于细胞外, 具有抗氧化、解毒等作用. 研究表明, Sep通过激活 HIF- $1 \alpha$, 干扰谷胱甘肽代谢, 增强氧化应激水平, 紊乱 线粒体功能, 诱导PASMC促增殖抗调亡; 低氧条件诱 导下, PASMC特异性敲除 $\mathrm{SeP}$ 小鼠能延缓HPH进程, 进 一步研究发现, 化合物血根碱能够特异性抑制Sep的表 达, 对 $\mathrm{PH}$ 具有治疗作用 ${ }^{[53]}$.

水通道蛋白1(aquaporin-1, AQP-1) 是细胞膜上运 输水分子以及 $\mathrm{O}_{2}, \mathrm{CO}_{2}, \mathrm{NO}$ 等气体的跨膜通道蛋白. 敲 除A q - 1 基因能破坏HIF- $1 \alpha$ 蛋白稳定性，有效抑制 PASMC过度增殖及PAEC功能紊乱, 减少缺氧性肺动 脉血管重构程度，降低肺动脉压，有效阻止HPH疾病 进程, 为 HPH的治疗提供潜在靶点和新思路 ${ }^{[54]}$. 


\section{3 治疗现状}

\section{$3.1 \quad$ 临床治疗局限性}

目前, 与 $\mathrm{HPH}$ 密切相关的3类PH暂无FDA批准治 疗方法 ${ }^{[55]}$, 辅助长程氧疗法是 3 类 $\mathrm{PH}$ 治疗的首要推 荐 $^{[2]}$, 然而长期氧疗耗时长, 只能改善部分肺泡缺氧, 小范围内降低肺动脉压力, 很多患者对于氧疗方式依 从性差, 导致治疗效果欠佳. 除氧疗外, 利尿剂、肺部 疾病的康复及潜在疾病的诊疗等辅助方法对 3 类 $\mathrm{PH}$ 的 治疗不容忽略 ${ }^{[56]}$.

1 类 $\mathrm{PH}$ (动脉型肺动脉高压) 靶向药物对 3 类 $\mathrm{PH}$ 的 治疗效果尚缺乏明确论证，包括5型磷酸二酯酶抑制 剂、内皮素受体拮抗剂、前列环素以及Rho激酶抑制 剂等. 大多数临床研究结果表明, 这些药物对 3 类 $\mathrm{PH}$ 的 症状、运动能力、血流动力学等指标无明显改善作 用. 例如, ARTEMIS-IPF试验结果显示, 内皮素受体拮 抗剂安立生坦无明显治疗作用, 且增加不良反应事件 的发生率, 导致试验提前终止 ${ }^{[57]}$; RISE IIP试验结果显 示, 利奥西呱(鸟苷酸环化酶激动剂)使用后, 患者死亡 率及严重不良事件发生率较高，试验提前终止以失败 告终 ${ }^{[58]}$; NSTAGE试验结果显示，与尼达尼布(酪氨酸 激酶抑制剂)单独用药相比，尼达尼布与西地那非( 5 型 磷酸二酯酶抑制剂)联合用药无明显作用优势 ${ }^{[59]}$. 然 而，最新一项大型临床试验结果显示，吸入曲前列环 素能对间质性肺疾病相关HPH预后具有明显改善作 用 ${ }^{[56]}$. 因此，需要更多的大型临床试验论证PH靶向药 物对HPH的疗效及安全性, 重点关注哪种类型疾病相 关的 $\mathrm{HPH}$ 能从中获益.

\section{2 挖掘天然产物治疗HPH}

天然产物是指动物、植物和微生物体内的化学成 分或其代谢产物, 作为药物发现的一个重要来源, 具有 来源丰富和结构新颖多样的特点. 已有多种天然产物或 其衍生物用于HPH治疗，发展前景良好，但目前大多处 在基础研究阶段, 距离转化成临床用药尚需大量研究.

褪黑素属于内源性吲哚胺类激素, 能透过血脑屏 障发挥生物学功能. 褪黑素本身及其级联代谢产物均
具有强大的抗氧化活性，对HPH实验动物的肺动脉血 管重构及肺动脉压升高具有显著抑制作用，潜在机制 可能与抑制PASMC的过度增殖及炎症反应，抑制内皮 激活相关 ${ }^{[60-63]}$; 原花青素B2(procyanidin B2) 属于黄酮 类天然产物，生物利用度高达 $90 \%$ 以上，能通过抑制 ROS过度生成, 增强抗氧化酶体系, 减少脂质过氧化物 的生成，进而发挥抗HPH大鼠肺动脉血管重构及肺动 脉压升高的作用 ${ }^{[64]}$; 小檗碱属于异喹啉生物碱, 能通过 上调骨形态发生蛋白 II 型受体(bone morphogenetic protein type II receptor, BMPR2)及下游分子P-smad1/5 的表达，下调TGF- $\beta$ 及下游分子P-smad $2 / 3$ 的表达，抑制 PASMC过度增殖、肺动脉血管重构，从而发挥抗 $\mathrm{HPH}$ 小鼠肺动脉血管重构、右心室收缩压升高及右心肥厚 的作用 ${ }^{[65]}$; 白藜芦醇属于非黄酮类多酚化合物，是sirtuin1(SIRT1)非特异性激活剂，通过三羧酸循环及氨基 酸、胆碱、亚油酸等代谢途径对低氧诱导的肺动脉高 压大鼠发挥治疗作用 ${ }^{[66]}$, 介导PI3K/Akt信号通路下调 精氨酸酶 II (arginase II)的表达，从而抑制PASMC增 殖 ${ }^{[67]}$, 缓解 $\mathrm{HPH}$ 进展. 药用植物在我国已有上千年历 史, 具有多治疗靶点特色, 中国人对药用植物拥有丰富 的临床经验, 目前研究报道己有多种中药提取物对 $\mathrm{HPH}$ 具有良好防治作用，如积雪草苷(五环三萜 类) ${ }^{[68]}$ 、异槲皮苷(黄酮醇类) ${ }^{[69]}$ 、灯盏花素(黄酮

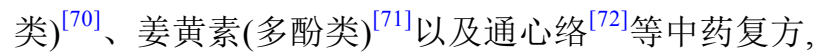
挖掘药用植物防治HPH 具有巨大潜力 ${ }^{[73]}$.

\section{4 展望}

$\mathrm{HPH}$ 疾病进展迅猛且预后不良，与多种心肺疾病 密切相关. 慢性炎症、氧化应激造成肺动脉血管重构 是重要病理生理学改变, 以其为靶点研发有效治疗药 物，有望实现延缓甚至逆转 HPH的疾病进展. HPH的 研究尚任重道远，发病分子机制的探索，天然产物、 小分子化合物等有效药物的管选与优化，基础科学向 临床诊疗的转化与应用等研究势在必行, 相信终将为 $\mathrm{HPH}$ 的防治带来曙光.

\section{参考文献}

1 Maarman G J, Chakafana G, Sliwa K. World Heart Day: a World Heart Federation communiqué on the future of basic sciences and translational 
medicine in global cardiovascular research. Am J Physiol Lung Cell Mol Physiol, 2020, 319: L545-L546

2 Group of Pulmonary embolism and Pulmonary Vascular Diseases, Chinese Society of Respiratory Medicine, Working Committee of Pulmonary embolism and Pulmonary vascular Diseases, Chinese Society of Respiratory Physicians, National Cooperation Group of Pulmonary Embolism and Pulmonary Vascular Diseases, et al. Chinese guidelines for diagnosis and treatment of pulmonary hypertension (2021 edition) (in Chinese). Natl Med J China, 2021, 101: 11-51 [中华医学会呼吸病学分会肺栓塞与肺血管病学组, 中国医师协会呼吸医师分会肺栓塞与肺血管病工 作委员会, 全国肺栓塞与肺血管病防治协作组, 等. 中国肺动脉高压诊断与治疗指南(2021版). 中华医学杂志, 2021, 101: 11-51]

3 Rowan S C, Keane M P, Gaine S, et al. Hypoxic pulmonary hypertension in chronic lung diseases: novel vasoconstrictor pathways. Lancet Respir Med, 2016, 4: 225-236

4 Feng E Z, Dai S G, Yang S Y. Research progress of hypoxic pulmomary artery hypertension (in Chinese). Chin J Lung Dis (Electr Ed), 2014, 7: 333-336 [冯恩志, 戴胜归, 杨生岳. 低氧性肺动脉高压研究进展. 中华肺部疾病杂志(电子版), 2014, 7: 333-336]

$5 \mathrm{Xu} \mathrm{K} \mathrm{Q,} \mathrm{Xia} \mathrm{S} \mathrm{J.} \mathrm{Mechanism} \mathrm{and} \mathrm{new} \mathrm{strategies} \mathrm{for} \mathrm{diagnosis} \mathrm{and} \mathrm{treatment} \mathrm{of} \mathrm{hypoxic} \mathrm{pulmonary} \mathrm{hypertension} \mathrm{(in} \mathrm{Chinese).} \mathrm{Chin} \mathrm{J} \mathrm{Lung} \mathrm{Dis}$ (Electr Ed), 2020, 13: 127-133 [徐康乔, 夏世金. 低氧性肺动脉高压发生机制与诊治新策略. 中华肺部疾病杂志(电子版), 2020, 13(02): 127133]

6 Stenmark K R, Fagan K A, Frid M G. Hypoxia-induced pulmonary vascular remodeling. Circ Res, 2006, 99: 675-691

7 Young J M, Williams D R, Thompson A A R. Thin air, thick vessels: historical and current perspectives on hypoxic pulmonary hypertension. Front Med, 2019, 6

8 Stevens T. Functional and molecular heterogeneity of pulmonary endothelial cells. Proc Am Thorac Soc, 2011, 8: 453-457

9 Xu W, Erzurum S C. Endothelial cell energy metabolism, proliferation, and apoptosis in pulmonary hypertension. Compr Physiol, 2011, 1: 357372

10 Smadja D M, Mauge L, Sanchez O, et al. Distinct patterns of circulating endothelial cells in pulmonary hypertension. Eur Respir J, 2010, 36: $1284-1293$

11 Tonelli A R, Haserodt S, Aytekin M, et al. Nitric oxide deficiency in pulmonary hypertension: Pathobiology and implications for therapy. Pulm Circ, 2013, 3: 20-30

12 Imamura M, Vitello A M, Limbird J N, et al. Endothelin-B receptor overexpression prevents hypoxic pulmonary hypertension in cirrhotic rats. Chest, 2005, 128: 580S-581S

13 Zhang $\mathrm{W}$, Zhu T, Wu W, et al. LOX-1 mediated phenotypic switching of pulmonary arterial smooth muscle cells contributes to hypoxic pulmonary hypertension. Eur J Pharmacol, 2018, 818: 84-95

14 Jie W, Guo J, Shen Z, et al. Contribution of myocardin in the hypoxia-induced phenotypic switching of rat pulmonary arterial smooth muscle cells. Exp Mol Pathol, 2010, 89: 301-306

15 Zhang J, Hu H, Palma N L, et al. Hypoxia-induced endothelial CX3CL1 triggers lung smooth muscle cell phenotypic switching and proliferative expansion. Am J Physiol Lung Cell Mol Physiol, 2012, 303: L912-L922

16 Zhang L Y, Gao B A. Relationship between pulmonary artery smooth muscle cells and mechanism of hypoxia-induced pulmonary vascular remodeling (in Chinese). Chin J Arterioscler, 2013, 21: 177-181 [张凌云, 高宝安. 肺动脉平滑肌细胞与低氧性肺血管重塑形成机制. 中国动 脉硬化杂志, 2013, 21: 177-181]

17 Lin M J, Leung G P H, Zhang W M, et al. Chronic hypoxia-induced upregulation of store-operated and receptor-operated Ca ${ }^{2+}$ channels in pulmonary arterial smooth muscle cells. Circ Res, 2004, 95: 496-505

18 Ball M K, Waypa G B, Mungai P T, et al. Regulation of hypoxia-induced pulmonary hypertension by vascular smooth muscle hypoxia-inducible factor-1 $\alpha$. Am J Respir Crit Care Med, 2014, 189: 314-324

19 Dai Z, Zhu M M, Peng Y, et al. Endothelial and smooth muscle cell interaction via FoxM1 signaling mediates vascular remodeling and pulmonary hypertension. Am J Respir Crit Care Med, 2018, 198: 788-802

20 Luo Y, Teng X, Zhang L, et al. CD146-HIF-1 $\alpha$ hypoxic reprogramming drives vascular remodeling and pulmonary arterial hypertension. Nat Commun, 2019, 10: 3551

21 Penaloza D, Arias-Stella J. The heart and pulmonary circulation at high altitudes. Circulation, 2007, 115: 1132-1146

22 Stenmark K R, Frid M G, Yeager M, et al. Targeting the adventitial microenvironment in pulmonary hypertension: a potential approach to therapy that considers epigenetic change. Pulm Circ, 2012, 2: 3-14

23 de Jesus Perez V A. Molecular pathogenesis and current pathology of pulmonary hypertension. Heart Fail Rev, 2016, 21: 239-257 
24 Wang D, Zhang H, Li M, et al. MicroRNA-124 controls the proliferative, migratory, and inflammatory phenotype of pulmonary vascular fibroblasts. Circ Res, 2014, 114: 67-78

25 Coen M, Gabbiani G, Bochaton-Piallat M L. Myofibroblast-mediated adventitial remodeling. Arterioscler Thromb Vasc Biol, 2011, 31: 23912396

26 Sheikh A Q, Lighthouse J K, Greif D M. Recapitulation of developing artery muscularization in pulmonary hypertension. Cell Rep, 2014, 6: 809817

27 Robinson C M, Neary R, Levendale A, et al. Hypoxia-induced DNA hypermethylation in human pulmonary fibroblasts is associated with Thy-1 promoter methylation and the development of a pro-fibrotic phenotype. Respir Res, 2012, 13: 74

28 Drexler E S, Bischoff J E, Slifka A J, et al. Stiffening of the extrapulmonary arteries from rats in chronic hypoxic pulmonary hypertension. J Res Natl Inst Stand Technol, 2008, 113: 239

29 El Kasmi K C, Pugliese S C, Riddle S R, et al. Adventitial fibroblasts induce a distinct proinflammatory/profibrotic macrophage phenotype in pulmonary hypertension. J Immunol, 2014, 193: 597-609

30 Li M, Riddle S R, Frid M G, et al. Emergence of fibroblasts with a proinflammatory epigenetically altered phenotype in severe hypoxic pulmonary hypertension. J Immunol, 2011, 187: 2711-2722

31 Frid M G, Brunetti J A, Burke D L, et al. Hypoxia-induced pulmonary vascular remodeling requires recruitment of circulating mesenchymal precursors of a monocyte/macrophage lineage. Am J Pathol, 2006, 168: 659-669

32 Eltzschig H K, Carmeliet P. Hypoxia and inflammation. N Engl J Med, 2011, 364: 656-665

33 Watts E R, Walmsley S R. Inflammation and hypoxia: HIF and PHD isoform selectivity. Trends Mol Med, 2019, 25: 33-46

34 Rudyk O, Aaronson P I. Redox regulation, oxidative stress, and inflammation in group 3 pulmonary hypertension. Adv Exp Med Biol, 2021, 1303: 209-241

35 Vergadi E, Chang M S, Lee C, et al. Early macrophage recruitment and alternative activation are critical for the later development of hypoxiainduced pulmonary hypertension. Circulation, 2011, 123: 1986-1995

36 Villegas L R, Kluck D, Field C, et al. Superoxide dismutase mimetic, MnTE-2-PyP, attenuates chronic hypoxia-induced pulmonary hypertension, pulmonary vascular remodeling, and activation of the NALP3 inflammasome. Antioxid Redox Signal, 2013, 18: 1753-1764

37 Smith K A, Schumacker P T. Sensors and signals: the role of reactive oxygen species in hypoxic pulmonary vasoconstriction. J Physiol, 2019, 597: 1033-1043

38 Waypa G B, Schumacker P T. Role for mitochondrial reactive oxygen species in hypoxic pulmonary vasoconstriction. Novartis Found Symp, 2006, 272: 176-192

39 Chen J, Wang Y X, Dong M Q, et al. Reoxygenation reverses hypoxic pulmonary arterial remodeling by inducing smooth muscle cell apoptosis via reactive oxygen species-mediated mitochondrial dysfunction. J Am Heart Assoc, 2017, 6

40 Zhang S, Yang T, Xu X, et al. Oxidative stress and nitric oxide signaling related biomarkers in patients with pulmonary hypertension: a case control study. BMC Pulm Med, 2015, 15: 50

41 Adesina S E, Kang B Y, Bijli K M, et al. Targeting mitochondrial reactive oxygen species to modulate hypoxia-induced pulmonary hypertension. Free Radic Biol Med, 2015, 87: 36-47

42 Jaitovich A, Jourd'Heuil D. A brief overview of nitric oxide and reactive oxygen species signaling in hypoxia-induced pulmonary hypertension. Adv Exp Med Biol, 2017, 967: 71-81

43 Veith C, Schermuly R T, Brandes R P, et al. Molecular mechanisms of hypoxia-inducible factor-induced pulmonary arterial smooth muscle cell alterations in pulmonary hypertension. J Physiol, 2016, 594: 1167-1177

44 Joppa P, Petrásová D, Stancák B, et al. Oxidative stress in patients with COPD and pulmonary hypertension. Wien Klin Wochenschr, 2007, 119: $428-434$

45 Satoh K, Nigro P, Berk B C. Oxidative stress and vascular smooth muscle cell growth: a mechanistic linkage by cyclophilin A. Antioxid Redox Signal, 2010, 12: 675-682

46 Reis G S, Augusto V S, Silveira A P C, et al. Oxidative-stress biomarkers in patients with pulmonary hypertension. Pulm Circ, 2013, 3: 856-861

47 Ornatowski W, Lu Q, Yegambaram M, et al. Complex interplay between autophagy and oxidative stress in the development of pulmonary disease. Redox Biol, 2020, 36: 101679

48 Wang C B, Li Z G, Peng B, et al. Research status of animal models of hypoxic pulmonary hypertension (in Chinese). J Emerg Trad Chin Med, 
2010, 19: 114-115 [王传博, 李泽庚, 彭波. 缺氧性肺动脉高压动物模型的研究现状. 中国中医急症, 2010, 19: 114-115]

49 Semenza G L. Hypoxia-inducible factors in physiology and medicine. Cell, 2012, 148: 399-408

50 Yan H, Zheng Q Y, Yang K, et al. Research progress on the role and mechanism of hypoxia-inducible factor in the pathogenesis of pulmonary hypertension (in Chinese). Chin J Tuberc Respir Dis, 2021, 44: 396-399 [颜涵, 郑秋玉, 杨凯, 等. 低氧诱导因子在肺动脉高压发病中的作用 及机制. 中华结核和呼吸杂志, 2021, 44: 396-399]

51 Dabral S, Muecke C, Valasarajan C, et al. A RASSF1A-HIF1 $\alpha$ loop drives Warburg effect in cancer and pulmonary hypertension. Nat Commun, 2019, 10: 2130

52 Cao Y, Zhang X, Wang L, et al. PFKFB3-mediated endothelial glycolysis promotes pulmonary hypertension. Proc Natl Acad Sci USA, 2019, 116: 13394-13403

53 Kikuchi N, Satoh K, Kurosawa R, et al. Selenoprotein P promotes the development of pulmonary arterial hypertension. Circulation, 2018, 138: $600-623$

54 Liu M, Liu Q, Pei Y, et al. Aqp-1 gene knockout attenuates hypoxic pulmonary hypertension of mice. Arterioscler Thromb Vasc Biol, 2019, 39: $48-62$

55 Galiè N, Humbert M, Vachiery J L, et al. 2015 ESC/ERS Guidelines for the diagnosis and treatment of pulmonary hypertension. Eur Heart J, 2016, 37: 67-119

56 Shioleno A M, Ruopp N F. Group 3 pulmonary hypertension: a review of diagnostics and clinical trials. Clin Chest Med, 2021, 42: 59-70

57 Raghu G, Behr J, Brown K K, et al. Treatment of idiopathic pulmonary fibrosis with ambrisentan: a parallel, randomized trial. Ann Intern Med, 2013, 158: 641

58 Nathan S D, Behr J, Collard H R, et al. Riociguat for idiopathic interstitial pneumonia-associated pulmonary hypertension (RISE-IIP): a randomised, placebo-controlled phase 2b study. Lancet Respir Med, 2019, 7: 780-790

59 Kolb M, Raghu G, Wells A U, et al. Nintedanib plus sildenafil in patients with idiopathic pulmonary fibrosis. N Engl J Med, 2018, 379: 17221731

60 Jin H, Wang Y, Zhou L, et al. Melatonin attenuates hypoxic pulmonary hypertension by inhibiting the inflammation and the proliferation of pulmonary arterial smooth muscle cells. J Pineal Res, 2014, 57: 442-450

61 Gonzaléz-Candia A, Candia A A, Figueroa E G, et al. Melatonin long-lasting beneficial effects on pulmonary vascular reactivity and redox balance in chronic hypoxic ovine neonates. J Pineal Res, 2020, 68: e12613

62 Zhang J, Lu X, Liu M, et al. Melatonin inhibits inflammasome-associated activation of endothelium and macrophages attenuating pulmonary arterial hypertension. Cardiovasc Res, 2020, 116: 2156-2169

63 Wang R, Pan J J, Li H, et al. Research progress of melatonin on pulmonary hypertension (in Chinese). Chin J Clin Pharmacol Ther, 2021, 26: 444-448 [王睿, 潘进进, 李华, 等. 褪黑素调节肺动脉高压的作用与机制研究进展. 中国临床药理学与治疗学, 2021, 26: 444-448]

64 Jin H, Liu M, Zhang X, et al. Grape seed procyanidin extract attenuates hypoxic pulmonary hypertension by inhibiting oxidative stress and pulmonary arterial smooth muscle cells proliferation. J Nutr Biochem, 2016, 36: 81-88

65 Chen M, Shen H, Zhu L, et al. Berberine attenuates hypoxia-induced pulmonary arterial hypertension via bone morphogenetic protein and transforming growth factor- $\beta$ signaling. J Cell Physiol, 2019, 234: 17482-17493

66 Sun X, Zhao B, Qu H, et al. Sera and lungs metabonomics reveals key metabolites of resveratrol protecting against PAH in rats. Biomed Pharmacother, 2021, 133: 110910

67 Chen B, Xue J, Meng X, et al. Resveratrol prevents hypoxia-induced arginase II expression and proliferation of human pulmonary artery smooth muscle cells via Akt-dependent signaling. Am J Physiol Lung Cell Mol Physiol, 2014, 307: L317-L325

68 Wang X, Cai X, Wang W, et al. Effect of asiaticoside on endothelial cells in hypoxia-induced pulmonary hypertension. Mol Med Report, 2017, 17: $2893-2900$

69 Li L, Dong P, Hou C, et al. Hydroxysafflor yellow A (HSYA) attenuates hypoxic pulmonary arterial remodelling and reverses right ventricular hypertrophy in rats. J Ethnopharmacol, 2016, 186: 224-233

70 Yang L, Zheng B X, Cheng D Y, et al. The effect of breviscapine on the pulmonary arterial pressure and the expression of Rho-kinase in pulmonary arterioles of hypoxic rats (in Chinese). Chin J Tuberculosis Respir Dis, 2008, 31: 826-830 [杨莉, 郑碧霞, 程德云, 等. 灯盏花素对低 氧大鼠肺动脉压和肺小动脉Rho激酶的影响. 中华结核和呼吸杂志, 2008, 31: 826-830]

71 Lin Q, Di F, Chen S X, et al. Effect of curcumin on pulmonary hypertension and wall collagen of pulmonary arterioles of chronic hypoxic 
hypercapnic rats (in Chinese). Chin J Appl Physiol, 2006, 22: 257-261 [林全, 狄枫, 陈少贤, 等. 姜黄素对慢性低氧高二氧化碳大鼠肺动脉高 压及肺动脉管壁胶原的影响. 中国应用生理学杂志, 2006, 22: 257-261]

72 Wang Y, Ma T T, Gao N N, et al. Effect of Tongxinluo on pulmonary hypertension and pulmonary vascular remodeling in rats exposed to a low pressure hypoxic environment. J Ethnopharmacol, 2016, 194: 668-673

73 Wang T, Hou J, Xiao W, et al. Chinese medicinal plants for the potential management of high-altitude pulmonary oedema and pulmonary hypertension. Pharm Biol, 2020, 58: 815-827

\title{
"Blue lips": research progress in pulmonary vascular remodeling in hypoxic pulmonary hypertension
}

\author{
WANG Rui, PAN JinJin, WANG DingYou, JI YuKe, LIU MingCheng, \\ JIN HaiFeng \& YUAN YuHui \\ Institute of Cancer Stem Cell, Dalian Medical University, Dalian 116044, China
}

Hypoxic pulmonary hypertension (HPH), characterized by increased pulmonary arterial pressure, is a complex and progressive disease with a wide spectrum of hypoxic etiologies and a challenging clinical problem with high disease prevalence. Mechanismbased treatment options for HPH are still lacking, and there is currently no cure for this devastating disease. Small pulmonary artery remodeling caused by chronic inflammation and aberrant oxidative stress under hypoxia is the typical pathological feature. This review summarizes the recent progress in pulmonary vascular remodeling research, including pathophysiological characteristics, pathophysiological pathogenesis, and treatment status and prospects, and provides ideas for the treatment of HPH.

pulmonary hypertension, hypoxia, pulmonary vascular remodeling, inflammation, oxidative stress

doi: $10.1360 / \mathrm{SSV}-2021-0432$ 